\title{
El aprendizaje gráfico de la recta tangente a través de la modelación de las secciones cónicas utilizando GeoGebra
}

\author{
The graphic learning of the tangent straight through the modeling of conical sections \\ using GeoGebra
}

\section{Aprendizagem gráfica do reto tangente através da modelagem das seções cônicas com GeoGebra}

\author{
Carlos Andrés Granados-Ortiz ${ }^{1}$ \\ Iván Andrés Padilla-Escorcia ${ }^{2}$
}

Recibido: abril de 2020

Aceptado: octubre de 2020

Para citar este artículo: Granados-Ortiz, C.A., Padilla-Escorcia, I.A. (2021). El aprendizaje gráfico de la recta tangente a través de la modelación de las secciones cónicas utilizando GeoGebra. Revista Científica, 40(1), 118-132. https://doi.org/10.14483/23448350.16137

\section{Resumen}

Esta investigación tuvo como objetivo fortalecer la modelación geométrica de la recta tangente en estudiantes de décimo grado mediante la utilización del software GeoGebra en una escuela pública de Barranquilla - Colombia para esto mediante una metodología cualitativa se aplicó una propuesta de tipo didáctico teniendo en cuenta las debilidades encontradas en los estudiantes partir de los resultados obtenidos en la etapa diagnóstica (pretest), concluyéndose que mediante la interacción de los estudiantes con este software los estudiantes potenciaron sus habilidades en el pensamiento geométrico, específicamente al modelar secciones cónicas en el software GeoGebra. Palabras clave: Modelación matemática, recta tangente, razonamiento, resolución de problemas, interpretación.

\begin{abstract}
This research aimed to strengthen the geometric modeling of the tangent line in tenth grade students by using the GeoGebra software in a public school in Barranquilla - Colombia. For this, through a qualitative methodology, a didactic proposal was applied taking into account the weaknesses found in the students from the results obtained in the diagnostic stage (pretest), concluding that through the interaction of the students with this software, the students enhanced their abilities in geometric thinking, specifically by modeling conic sections in the GeoGebra software. Keywords: Math's modeling, tangent line, reasoning, solving problem, interpretation.
\end{abstract}

1. Universidad del Atlántico. Barranquilla, Colombia. cagranados@mail.uniatlantico.edu.co

2. Universidad del Atlántico. Barranquilla, Colombia. iapadilla@mail.uniatlantico.edu.co 


\section{Resumo}

Esta pesquisa teve como objetivo fortalecer a modelagem geométrica da linha tangente em alunos da décima série, utilizando o software GeoGebra em uma escola pública de Barranquilla - Colômbia. Para tanto, por meio de uma metodologia qualitativa, foi aplicada uma proposta didática levando em consideração as fragilidades encontradas nos alunos a partir dos resultados obtidos na etapa de diagnóstico (pré-teste), concluindo que através da interação dos alunos com este software, os alunos potencializaram suas habilidades em pensamento geométrico, especificamente modelando seções cônicas no software GeoGebra.

Palavras-chaves: Modelação matemática, linha tangente, raciocínio, resolução de problemas, interpretação.

\section{Introducción}

Con el pasar de los años, la inserción de la tecnología se ha convertido en un elemento clave en las aulas de clases, en el profesorado, pero sobre todo en las motivaciones escolares del estudiantado en general, siendo que la integración de estos elementos proporciona nuevas oportunidades y entornos de aprendizaje. En términos específicos de la enseñanza de las matemáticas, softwares como GeoGebra, Cabri, Matlab, entre otros, se vislumbran en su implementación como elementos de orden tecnológico con mayor aplicación en las aulas de clase debido a que promueven en el estudiantado el desarrollo de habilidades matemáticas y niveles de entendimiento y abstracción como la visualización y exploración de contenidos de las matemáticas a través de entornos multimedia (Barahona, Barrera, Vaca e Hidalgo, 2015).

Ahora bien, lograr que estas herramientas sean integradas en los salones de clases en la enseñanza de contenidos de matemáticas, depende en parte del interés y el deseo del profesorado para crear y generar conocimientos por medio de la elaboración de clases dinámicas que estimulen el aprendizaje continuo, colaborativo y cooperativo de los alumnos. Así mismo, teniendo en cuenta que las mismas ofrecen la oportunidad de permitir nuevas y mejores formas de llevar a cabo el aprendizaje en los estudiantes mediante la exploración y manipulación de softwares educativos, sacar sus propias conclusiones es una de las principales características como resultado o producto que ofrecen el uso de este tipo de herramientas en el aula. (Arias Ronald, 2013).

Lo anterior, atendiendo la necesidad de la constante búsqueda de nuevas estrategias y métodos de enseñanza en el profesor, el cual a través de su trabajo propicia una personalización de la matemática mediante la creación de situaciones aplicables en el contexto real en donde se evidencien los conocimientos matemáticos abordados. Debido a que en la solución de problemas se encuentra inmersa como un elemento clave en el aprendizaje del alumnado, siendo que la intervención del profesor es de vital importancia en dicho proceso en las distintas reflexiones debe llevar a cabo con respecto a qué tipo de problemas o situaciones del diario vivir fomentan el desarrollo cognitivo de competencias matemáticas que permitan a los estudiantes aplicar los contenidos que aprenden (Zaldívar, Medina y Kakes, 2018).

Ahora, de acuerdo con Sáenz, Patiño y Robles (2017), es en el pensamiento geométrico, donde se presentan más dificultades y obstáculos en el estudiantado, especialmente en la interpretación de modelos gráficos aplicados a situaciones del diario vivir. Siendo una de las principales razones que conlleva a lo antes en mención que este pensamiento es el que menos prioridad suelen brindar en los procesos de enseñanza - aprendizaje el profesorado en general, limitando a los estudiantes a desarrollar habilidades de análisis y razonamiento en el modelado de situaciones problemáticas de carácter geométrico. Así mismo, el MEN (1998) en los Estándares Básicos de Competencia, afirma que la abstracción y rigurosidad que ofrece la geometría hacen compleja tanto la enseñanza como su aprendizaje, lo que denota la relevancia no solamente de atender este tipo de pensamiento en las aulas de clase, sino que partiendo que la dificultad que genera el mismo, se requiere de la formación 
del profesorado en el uso de recursos que permitan llevar a cabo una enseñanza más fluida, esto es la cualificación constante de los docentes en todos los ámbitos posibles (Padilla, Valbuena y Rodríguez, 2018).

Es así, como en los últimos años se ha vislumbrando en las escuelas la utilización del software GeoGebra en la enseñanza de las matemáticas escolares, específicamente por su aplicabilidad en el componente netamente geométrico, entendiéndose que a través de este software es posible que los estudiantes lleven a cabo manipulaciones dinámicas del entorno, registros de representación de un mismo objeto matemático que integra la geometría y que ayuda a visualizar problemas de la vida cotidiana, además es de carácter amigable para los estudiantes (Gruszycki et al, 2012; Córdoba, Ruíz y Rendón, 2015). Siendo que la interacción entre el profesor - estudiante-GeoGebra es uno de los elementos claves de éxito de esta herramienta, aparte que es un software de aplicación libre con código abierto ideal para la creación de applet interactivos lo que en el contexto escolar facilita su accesibilidad tanto para las escuelas, profesorado y estudiantes.

De este modo, este proyecto centra su atención en una escuela de carácter oficial en BarranquiIla - Colombia, en esta los estudiantes de décimo grado presenten dificultades en la interpretación de gráficas, modelación de situaciones contextuales y escasa aplicación de la geometría en la solución de estos problemas antes en mención. Lo anterior debido a la poca profundización en cuanto a teorías, contenidos, propiedades y principios matemáticos que se Ilevan a cabo en el salón de clases por parte del profesor que dicta matemáticas en dicho año escolar, lo impide que los estudiantes asuman un rol activo en el desarrollo de sus conocimientos.

Lo cual se confirmó a través de la observación, con respecto a que los profesores del grado décimo de la institución educativa no hacen uso de herramientas tecnológicas que promuevan el aprendizaje cooperativo de los estudiantes, lo que denota en que estos con conocimientos básicos de las TIC en educación matemática aplicado a la escuelas, lo cual es grave si se considera que esta es una de las competencias al día de hoy de mayor exigencia y que está dentro de las competencias que propone el MEN y que todo profesor debe tener; definiéndose estas en cuatro categorías: (i) competencia tecnológica la cual es la capacidad para seleccionar y utilizar de forma pertinente, responsable y eficiente variedad de herramientas tecnológicas de acuerdo a los principios que las rigen, la forma de combinarlas y las licencias que las amparan; (ii) competencia comunicativa, que se define como capacidad de relacionar espacios virtuales y audiovisuales a través de los medios utilizando diversos lenguajes sincrónicos y asincrónicos; (iii) competencia pedagógica, que se define como la capacidad de utilizar las TIC para fortalecer los procesos de enseñanza y aprendizaje en el estudiantado partiendo de las fortalezas y debilidades que ofrece la incorporación de estas herramientas en la formación integral de los estudiantes y en su propio desarrollo profesional como seres integrales; (iv) competencia investigativa, la cual es la capacidad del profesor para hacer uso de las TIC en la generación de nuevos conocimientos (MEN, 2013).

Así y de acuerdo a las necesidades que se evidencian en dicha institución con respecto al uso de la tecnología, es influyente las quejas e inconformidades por parte del alumnado con respecto al método de enseñanza tradicionales que proponen los profesores (Padilla y Mayoral, 2020), esto pues, teniendo en cuenta las competencias digitales que debe tener el profesorado según el MEN se hace necesario que las escuelas no solo analicen las prácticas de sus profesores, sino que construyan planes de mejora que permitan la cualificación de los mismos. De este modo, esta investigación tiene como objetivo fortalecer la modelación geométrica de la recta tangente en estudiantes de décimo grado a través de la mediación del software GeoGebra en una escuela pública de Barranquilla - Colombia. 


\section{Modelación y GeoGebra en Educación Matemática}

Es en el año 1998 cuando en Colombia se comienza a hablar de la modelación en el contexto educativo, específicamente en el pensamiento matemático, a través de los lineamientos curriculares de ese entonces se estableció que para llevar al desarrollo del mismo, este debía ir encaminado a cinco procesos fundamentales: la elaboración, comparación y ejercitación de procedimientos, el razonamiento, la resolución y planteamiento de problemas, la comunicación y la modelación (proceso de interés de este proyecto) (MEN, 1998).

De acuerdo con el MEN (1998) el punto de partida de la modelación está en la identificación de una situación problemática real, que posteriormente debe ser simplificada, idealizada, estructurada y sujeta a condiciones y suposiciones que requieren de precisión de acuerdo con los intereses del problema indagado que conduzcan a la formulación de este, de manera que puedan ser aplicados en el aula de clases. Así la modelación se entiende como un proceso completo que conduce desde la situación de una problemática real original hasta la construcción de un modelo matemático basado en la misma problemática y que se fundamente en una actividad científica que se encargue de resolver problemas de otras ciencias o para avanzar en una teoría que por lo general es de tipo aplicado por medio dichos concepto matemáticos y que despierta interés y motivación por el aprendizaje de las matemáticas debido a la aplicabilidad y relación que esta área del conocimiento evidencia con la vida real (Villa y Ruíz, 2009).

Así mismo, al incorporarse en las clases de matemáticas la modelación ofrece múltiples ventajas debido a que permite establecer relaciones entre las matemáticas y la "realidad" asociada a los contextos extraescolares (Villa, Rojas y Cuartas, 2010). Lo anterior conlleva a pensar, que es este proceso matemático, clave en el desarrollo de habilidades que permitan no sólo contextualizar a los estudiantes con situaciones de su diario vivir, sino modelar estas mismas por medio de patrones matemáticos, siendo evidente que en la escuela se requiere formar estudiantes que piensen de manera científica y crítica en la solución de problemas, lo que se evidencia en lo afirmado por (Verschaffel y De Corte, 1997; MaaB, 2007 y Villa, 2015) con respecto a que la poca implementación de la modelación matemática en las escuelas ha ocupado un lugar relevante dentro de las agendas de investigación a nivel internacional; esto dado el interés que representa la manera en que los profesores implementan o no la modelación matemática en la enseñanza.

por otra parte, Villa, González y Carmona (2018) afirman que son las TIC un elemento que cumple papel clave en la obtención y análisis de datos que producen, validan y analizan modelos matemáticos construidos con base a una situación problemática que se derive de contenidos matemáticos como las funciones, derivadas, ecuaciones diferenciales, entre otros. Siendo así, GeoGebra, un software matemático que cuenta con distintas herramientas y comandos inmersos que fomenta al desarrollo de la modelación matemática.

De este modo, diversas investigaciones a nivel nacional e internacional se han realizado con el objetivo de a través de esta herramienta facilitar la enseñanza de las matemáticas, fortalecer el componente de la modelación y fomentar al trabajo en grupo y colaborativo utilizando la tecnología, específicamente el software GeoGebra. En ese orden de ideas, Bejarano (2017) a nivel internacional realizaron una investigación titulada; "modelización matemática y GeoGebra en el estudio de funciones. Una experiencia con estudiantes de ingeniería", cuyo objetivo estuvo enmarcado en analizar las producciones de los estudiantes, relacionadas con el concepto de función y sus propiedades, mediante la implementación de tareas para resolver problemas de modelización matemática; incluyendo fenómenos y situaciones relacionadas con la ingeniería. Para esto, se hizo foco en la búsqueda y exploración de situaciones problemas contextualizadas que abarcaran los distintos tipos 
de funciones (polinómicas, exponenciales y trigonométricas) y que reflejaran la modelación matemática visualizadas en el software GeoGebra en el desarrollo del pensamiento variacional, para construir, resolver, inferir, validar y comunicar resultados de los modelos. Concluyendo en la misma que los estudiantes comprendieron definiciones matemáticas (funciones) y las vincularon a sus respectivos gráficos mediante la modelización matemática apoyados en GeoGebra que les permitió simular el comportamiento de fenómenos contextualizados que variantes de acuerdo con los parámetros presentes en cada modelo construido. Además, esta investigación permitió contribuir a que los estudiantes aplicaran los conceptos que tenían presentes sobre funciones para analizar, modelar y simular fenómenos planteados al inicial (modelado) pero en contextos diferentes.

Ahora bien, en el contexto nacional Flórez y Yemail (2017) realizaron el proyecto titulado: "modelación y simulación con GeoGebra: una experiencia en el estudio de situaciones con medidas de área y volumen", el objetivo de estudio de esta consistía en realizar procesos de modelación y simulación para la solución de situaciones problema relacionadas con medidas de área y volumen a través de funciones, utilizando como herramienta interactiva el software GeoGebra. Para esto, se diseñó una unidad didáctica que involucrara los procesos de modelación y simulación de manera interactiva por medio de GeoGebra en los estudiantes de noveno grado a través de una situación problemática contextualizada en los contenidos de área y volumen que pudiera ser modelada matemáticamente mediante el tema de funciones. Concluyendo que este trabajo permitió dar muestra de las ventajas que ofrece el desarrollo de la modelación matemática apoyado en el software GeoGebra para la construcción de modelos de con funciones para fortalecer el aprendizaje de conceptos matemáticos básicos como lo es el área y volumen. Aparte este trabajo contribuyó a resaltar la relevancia del estudio de la modelación y la simulación, como procesos clave para comprender las relaciones existentes entre las matemáticas y los contextos reales.

Finalmente, se exploró también a nivel nacional la investigación realizada por Pabón, Nieto y Gómez (2015) titulada: "modelación matemática y GeoGebra en el desarrollo de competencias en jóvenes investigadores" la cual tuvo como objetivo analizar las competencias de jóvenes investigadores utilizando software GeoGebra, siendo el propósito de la misma mostrar la importancia de fomentar en el estudiantado el manejo de esta herramienta como facilitadora en el desarrollo de competencias matemáticas como la visualización y simulación de situaciones reales de manera dinámica e interactiva; a parte la necesidad que conlleva la incorporación a los planes curriculares de estos softwares interactivos en la enseñanza de los contenidos de matemáticas. Para esto a los estudiantes se les realizó una presentación en la cual se les dio a conocer los conceptos claves sobre la modelación y su importancia en la solución de problemas, inicialmente sin el uso del software GeoGebra, posterior a esto se les presentó al grupo la explicación de las herramientas básicas de GEOGEBRA para que realizaran un ejercicio que les permitiera apropiarse del manejo del software, finalmente modelar una función matemática e interpretar los resultados de esta. Concluyendo que los jóvenes investigadores tienen potenciales en el desarrollo del pensamiento geométrico a partir de la construcción de gráficas y modelado de situaciones de la vida real a través del software GeoGebra.

\section{Método}

El enfoque utilizado para esta investigación es de tipo cualitativo debido a que se fundamenta un proceso inductivo que explora, describe y genera perspectivas teóricas que van de lo particular a lo general (Hernández- Sampieri, 2014). En este contexto se busca la recolección de los datos a partir de cada perspectiva y puntos de vista de los participantes. Así mismo las interacciones entre individuos y grupos, en donde el investigador entrevista 
a una persona, analiza los datos que obtuvo y saca algunas conclusiones; posteriormente, entrevista a otra persona, analiza esta nueva información y revisa sus resultados y conclusiones; del mismo modo, analiza más entrevistas para comprender lo que busca, es decir, procede caso por caso, dato por dato, hasta llegar a una perspectiva más general de la información.

Por otra parte, el diseño de investigación para el desarrollo de esta propuesta es de hermenéutico-interpretativo (Hernández - Sampieri, 2014) y consta de cinco fases en su diseño, la primera es la elección del tema que fue seleccionado a partir del campo laboral de investigación que es el aula, ya que se observó dificultades al momento de identificar la tangente en cualquier situación cotidiana; la segunda fase consta en identificar las características necesarias del recurso TIC para el desarrollo de la modelación de la interpretación geométrica de la tangente, para saber qué herramientas servirán de apoyo al momento de diseñar la propuesta; la tercera fase sigue con diseñar actividades a través de recursos TIC que faciliten la visualización para el análisis e interpretación de la tangente; la cuarta fase continúa con implementar el recurso TIC al proceso de enseñanza-aprendizaje para la interpretación de la tangente; la última fase es evaluar el aprendizaje de los estudiantes donde ellos interpretan geométricamente la tangente y de esa manera validar la propuesta didáctica por la presente investigación.

De igual manera, la población seleccionada para la aplicación de la propuesta didáctica: interpreta geométricamente la tangente, haciendo uso del software GeoGebra, es de un grupo de estudiantes de décimo grado que pertenecen a una institución de carácter oficial de Barranquilla ubicado en el suroccidente de la ciudad, en una zona comercial de extracto medio. La escogencia de la muestra se dio bajo los criterios de una muestra no probabilística o muestra dirigida, con un tipo de muestreo 'los sujetos-tipos', esta utilizada en estudios cualitativos donde el objetivo es la riqueza, profundidad y calidad de la información, y no la cantidad y estandarización, en este caso los estudiantes debían tener dificultades en la interpretación geométrica de la recta tangente (Hernández - Sampieri, Fernández y Baptista, 2007).

\section{Fases de la investigación:}

\section{Elección de los participantes}

La población que se selecciona para la aplicación de la propuesta didáctica: Interpreta geométricamente la tangente, haciendo uso del software GeoGebra, es de un grupo de estudiantes de décimo grado que pertenecen a una institución educativa distrital de la ciudad de barranquilla ubicado en el suroccidente de la ciudad, en una zona comercial de extracto medio. Para escoger la muestra, el curso escogido es de décimo $C$, este grupo será evaluado de manera individual después de haberse aplicado la propuesta acompañados por el docente de matemáticas a cargo. La totalidad de estudiantes en el grado son de 35 estudiantes, lo cual, se seleccionaron nueve estudiantes, para esto se tuvo como referencia (Hernández - Sampieri, Fernández y Baptista, 2007), se seleccionó una muestra no probabilística o muestra dirigida, con un tipo de muestreo 'los sujetos-tipos' es utilizada en estudios cualitativos donde el objetivo es la riqueza, profundidad y calidad de la información, y no la cantidad y estandarización.

\section{Elaboración y aplicación de la propuesta}

Esta propuesta es planteada para brindar un apoyo que permita a docentes, estudiantes o cualquier ente se interese en promover el desarrollo de la modelación matemática a través del proceso de aprendizaje; la interpretación geométrica de la recta tangente a través de las secciones cónicas por medio de actividades planteadas en el software GeoGebra; este programa facilitara a los estudiantes a identificar que es una recta tangente en una curva 
determinada con ciertos puntos establecidos y a su vez interpretar su gráfico.

\section{Técnicas en la recolección de datos}

Las técnicas de recolección de la presente investigación se llevaron a cabo, mediante las siguientes:

\section{Encuesta}

En la encuesta se realizaron una serie de preguntas sobre uno o varios temas a una muestra de personas seleccionadas siguiendo una serie de reglas científicas que hacen que esa muestra sea, en su conjunto, representativa de la población general de la que procede.

\section{Entrevista}

Para el presente proyecto de investigación esta técnica se utilizó para interactuar de manera abierta haciendo uso del tipo de entrevista no estructurada, donde indica que la información que se obtiene de ella es el resultado de la construcción simultánea a partir de las respuestas del entrevistado con los estudiantes y el profesor, en el grado décimo C, se escogieron 3 estudiantes apoyándonos de la entrevista de desarrollo o de seguimiento la cual describe el proceso de evolución de un aspecto determinado dentro del contexto de estudio, las preguntas de la entrevista ya estarán brevemente escritas y a medida que fluya la conversación se irá respondiendo una a una, esta entrevista se realizará antes y después de desarrollar la propuesta didáctica.

\section{Test inicial}

El objetivo de la prueba inicial consistió en determinar el estado de conocimiento en la cual se encuentra el estudiante, es decir, los conocimientos previos que maneja el estudiante en este caso sobre la tangente, la línea recta, sus propiedades, donde se aplica y que concepciones tienen y las dificultades que presentan de la temática

\section{Test final}

El objetivo de este test consistía en verificar posteriormente a la aplicación de la propuesta que los estudiantes de décimo grado:

- Graficaran en el software GeoGebra.

- Interpretaran la gráfica con GeoGebra.

- Calcularan la ecuación de la recta tangente en determinado punto con GeoGebra.

- Diferenciaran la variación que ocurre entre las curvas haciendo uso de GeoGebra.

Para la elaboración de la propuesta se tuvo en cuenta los siguientes actos pedagógicos:

\section{Etapa Motivacional}

En esta etapa se introdujo en los estudiantes pequeñas nociones o indicios de línea recta, plateando preguntas tales como: ¿Qué es una línea recta? ¿Dónde se aplica la línea recta? O ¿Para qué sirve?, con el fin de escuchar las opiniones de los(a) estudiantes e interactuar con ellos(a) puesto que esto es importante para el desarrollo de las actividades.

Se pretende con el planteamiento de estas actividades Ilamar la atención de los(a) estudiantes, quienes mostraron interés por lo tecnológico durante las observaciones realizadas, además de esto, la etapa consistía en estar en una clase presencial donde estuvieran los investigadores explicando su uso para que sirven y la importancia que tiene en la vida cotidiana.

Este paso es muy importante porque se le muestra a los(a) estudiantes lo interesante de la línea recta, sus apariciones en la cotidianidad, curiosidades y aplicabilidad de estas.

\section{Etapa Conceptual}

Para esta etapa se propone dos sub-etapas

Presencial: En la cual se dictan las clases tradicionales presenciales, en el aula desarrollando la temática de la recta, sus propiedades, sus 
definiciones, propiedades y gráficas, entre otras, los estudiantes tendrán una mayor idea para posterior mente realizar las actividades.

Virtual: En sala de computación de la institución se instaló en software GeoGebra que le sirve a los estudiantes como apoyo para el desarrollo de las clases presenciales, los investigadores a cargo que desarrollaron la temática a cargo donde se dio inicio a la primera actividad de la propuesta en la cual iban a conocer Geogebra y empezar a desarrollar los pasos prescritos en la actividad. Además de esto se proponen actividades que ayuden al afianzamiento de los conocimientos aprendidos articulados en la propuesta, en el desarrollo de la primera actividad después de finalizar la presentación del software, empezando la interacción (Fig 1).

\section{Etapa de Aplicabilidad}

En esta etapa se proponen actividades en el software de la temática tratada en la etapa anterior se hablaba de GeoGebra la cual hará construcciones utilizando las definiciones de la línea recta, recta tangente a una curva, propiedades, ecuaciones, entre otras, también demostraran su destrezas y capacidad para razonar visualizar y plantear soluciones a distintas situaciones problema que se plantean, los estudiantes (Fig 2).

\section{Etapa de Evaluación}

En esta etapa se realiza una retroalimentación y autorreflexión de los contenidos aprendidos, de manera que los estudiantes nos expresen sus experiencias y lo que lograron desarrollar, todo esto a través de comentarios y opiniones que realicen durante la intervención de la propuesta, por otro lado, se aplica el Test Final como medio de evaluación, también se realiza una evaluación de forma virtual con el fin de analizar lo generado por la propuesta pedagógica.

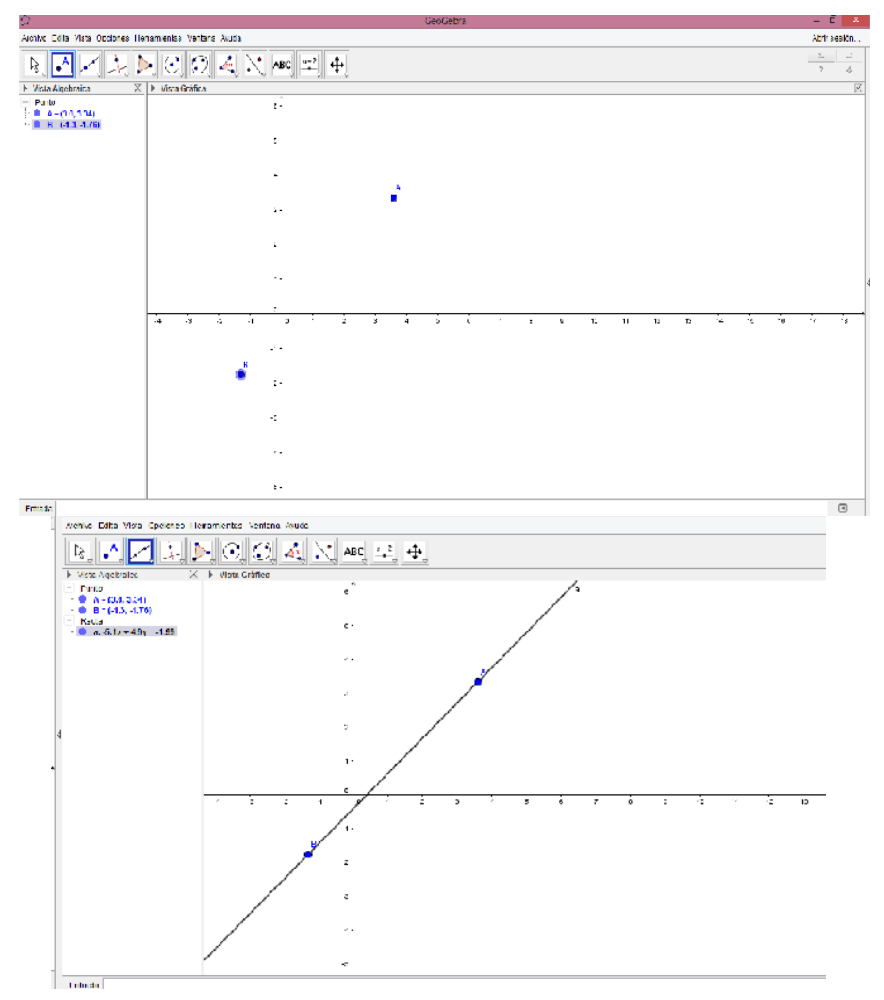

Figura 1: Desarrollando de la primera actividad.

Fuente: Los autores. 
Además de esto se aplica una entrevista final para conocer la opinión de los(a) estudiantes acerca las actividades realizadas, su nivel de satisfacción, sugerencias.

En consecuencia, a los(a) estudiantes de la institución educativa distrital se les realizó una evaluación virtual y una evaluación escrita lo cual fue aplicada después de la evaluación virtual, en esta fase mediante el uso de GeoGebra se les indico a los estudiantes los pasos a seguir y dicha evaluación iba a ser enviada por correo electrónico (Fig. 3)

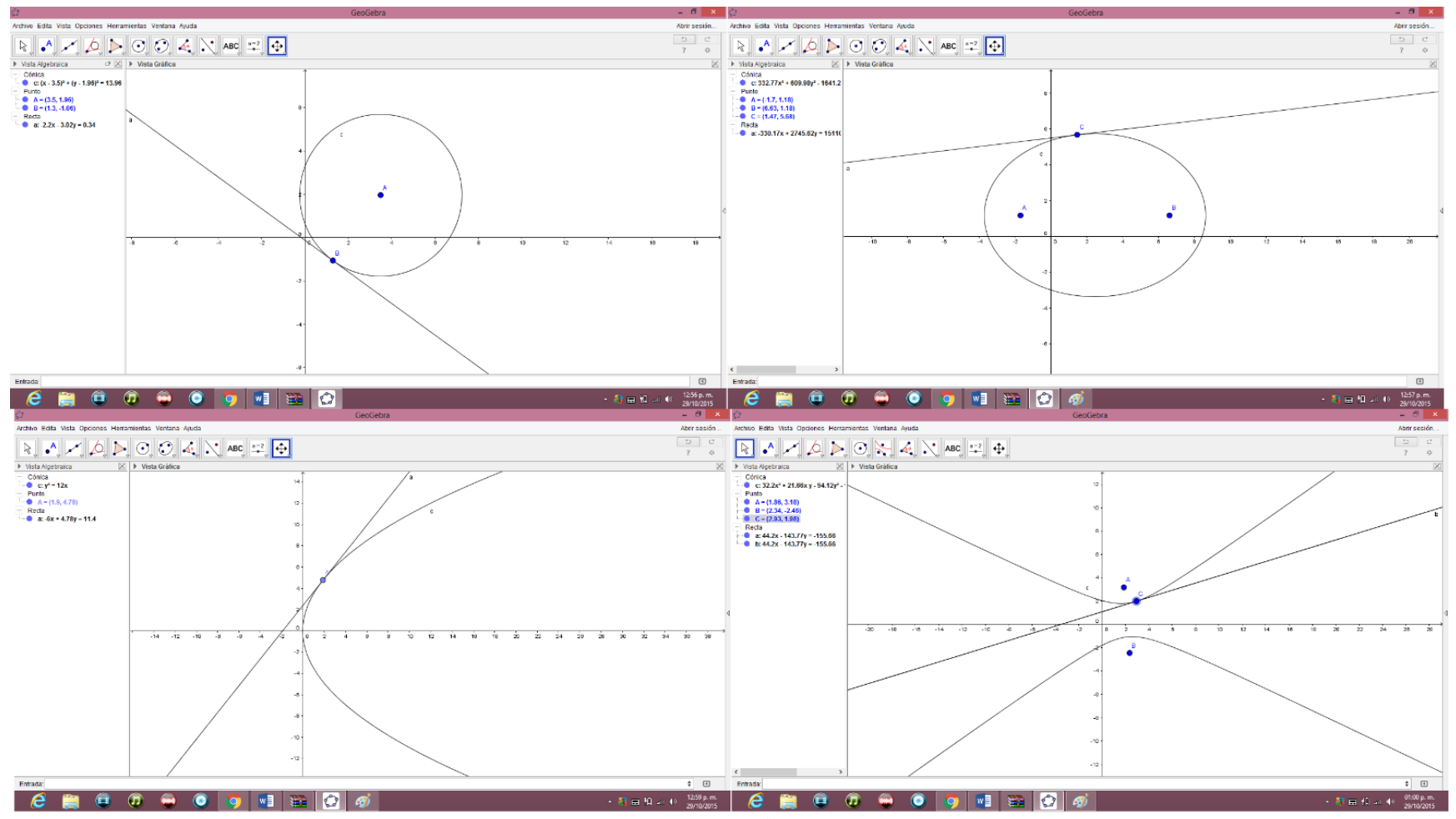

Figura 2. Rectas tangente a las secciones cónicas.

Fuente: Los autores.

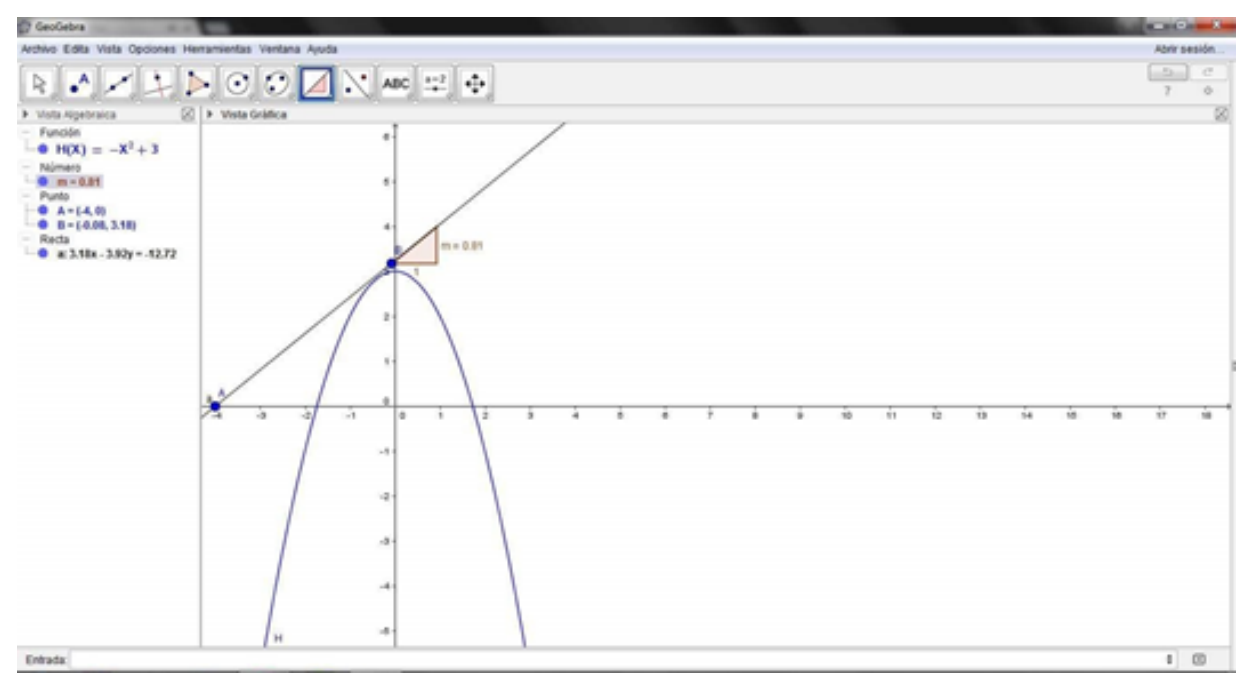

Figura 3. Evaluación virtual de un estudiante.

Fuente: Los autores. 


\section{Resultados y discusión}

En relación con los objetivos del presente artículo, se realizó un estudio, utilizado un test inicial y un test final con la muestra seleccionada, para determinar la implementación de la propuesta pedagógica es efectiva para la interpretación geométrica de la recta tangente.

En la figura 4, podemos observar que los estudiantes poseen dificultades al momento de hallar o resolver problemas donde se involucre la línea recta. De manera general solo se respondieron las preguntas correctamente en un 5\%, es decir que hay necesidad de aplicar otras estrategias para mejorar el proceso de enseñanza-aprendizaje de este concepto.

Ahora procederemos a realizar el análisis las entrevistas realizadas a los estudiantes y al docente de matemáticas, esta entrevista se realizó de manera no estructurada, el cual permite que los entrevistados den su opinión con respecto a una pregunta planteada y no limitarse a unas respuestas específicas.

A continuación, se realiza el análisis del Test Final, después de haberse aplicado la propuesta, los resultados están descritos en la tabla 3.

Tabla 1: Análisis del test inicial.

Preguntas

Descripción

Esta pregunta corresponda a uno de los

¿Podría graficar en el plano cartesiano dos puntos y unirlos?

Teniendo en cuenta el punto anterior, ique se forma al unir los dos puntos?

De la ecuación
$x+2 y+3=0$, ¿Qué forma
obtiene la recta al
despejar la ' $y$ '?

De la ecuación obtiene la recta al despejar la ' $y$ '? postulados de Euclides, donde menciona que, al unir dos puntos cualesquiera, formará una línea recta, esto con el fin de conocer si los estudiantes tienen conocimiento de este mismo.

Esta pregunta está ligada con la pregunta anterior, porque aquellos estudiantes que tengan el concepto Euclidiano, al unir los dos puntos observaran la figura geométrica que se está formando.

Esta pregunta está enfocada a algunos conceptos fundamentales de la geometría analítica plana, teniendo en cuenta el libro de Lehmann (1989), menciona la diferencia entre una línea recta de la forma general y de la forma canónica, además de por qué y cuándo se debe escribir de una manera u otra.

\section{Análisis}

Teniendo en cuenta la descripción de esta pregunta, se pudo observar que solamente dos estudiantes respondieron correctamente esta misma, y este concepto es fundamental al momento de enseñarle a los estudiantes la línea recta analíticamente, tal como lo menciona Hitt (2003), una de las principales dificultades el cual no le permite a los estudiantes entender el concepto analítico de la línea recta, parte desde la fundamentación teórica de la geometría en el plano unidimensional.

Teniendo en cuenta la descripción y los resultados de la pregunta anterior, solo un estudiante respondió correctamente este punto, algunos manifestaban no estar seguro de su respuesta, porque no habían entendido cuando el docente explicó esta temática. Font (1994), menciona la importancia que tiene el docente de matemáticas dentro del aula al momento de impartir una clase y la motivación extrínseca que reciba el estudiante, esto para ayudarlo en su proceso de aprendizaje y así facilitar el proceso de enseñanza.

Teniendo él cuenta la descripción de la pregunta, se pudo observar que ningún estudiante la respondió correctamente, al momento de revisar el test inicial, se evidenció que cuatro estudiantes despejaron erróneamente la ecuación y los demás no las respondieron manifestando que no sabían. El ministerio de educación nacional (2016), en una de las evidencias de sus DBA menciona que el estudiante debe de ser capaz de convertir una ecuación de su forma general a su forma canónica, es decir, que debemos desarrollar habilidades en el estudiante que le permitan resolver este tipo de problemas. 
Teniendo en cuenta los puntos $\quad A(0,2) \quad B(3,1)$ ¿cuál es su pendiente?

\section{¿S y $R$ son perpendiculares?}

\section{¿J y I son paralelas?}

Esta pregunta, tiene como finalidad identificar si el estudiante puede hallar la pendiente de una línea recta a través de dos puntos. Recordemos que la pendiente es muy importante este tema, porque con ella puede conocer la variación de mis datos e incluso saber si mis datos son crecientes o decrecientes.

Esta pregunta está enfocada en conocer si los estudiantes habían entendido este concepto, pero recordemos que, en la geometría analítica, este concepto va ligado a la pendiente de una recta.

Esta pregunta está enfocada en conocer si los estudiantes habían entendido este concepto, pero recordemos que, en la geometría analítica, este concepto va ligado a la pendiente de una recta, también, se podía observar si los estudiantes sabían que una línea recta tiene una familia de líneas rectas.
Teniendo en cuenta la descripción de la pregunta, ningún estudiante respondió correctamente este ejercicio, los estudiantes seguían manifestando que el tema si lo habían abordado, pero no lo habían entendido.

Todos los estudiantes respondieron que, si eran perpendicular, pero no sabían cómo hallar la línea recta perpendicular, tal como lo menciona Lehmann (1989) en su libro, dos rectas son perpendiculares si el producto de sus pendientes es negativo y teniendo en cuenta el análisis de la pregunta anterior, los estudiantes no tenían un claro concepto de la pendiente.

Todos los estudiantes respondieron que las rectas son paralelas, pero no sabían cómo hallar la recta. El concepto de rectas paralelas puede ser más sencillo de entender ya que las pendientes son iguales solo varia el punto de corte con el eje y, tal como lo menciona Ramírez, Oktac y García (2006), explicar que dos rectas son paralelas es sencillo, cualquier docente de matemáticas preparado lo podría hacer, el reto es explicarles a los estudiantes hallar rectas perpendiculares.

Fuente: Elaboración propia con base a los resultados obtenidos en el test inicial.

\section{Test inicial}

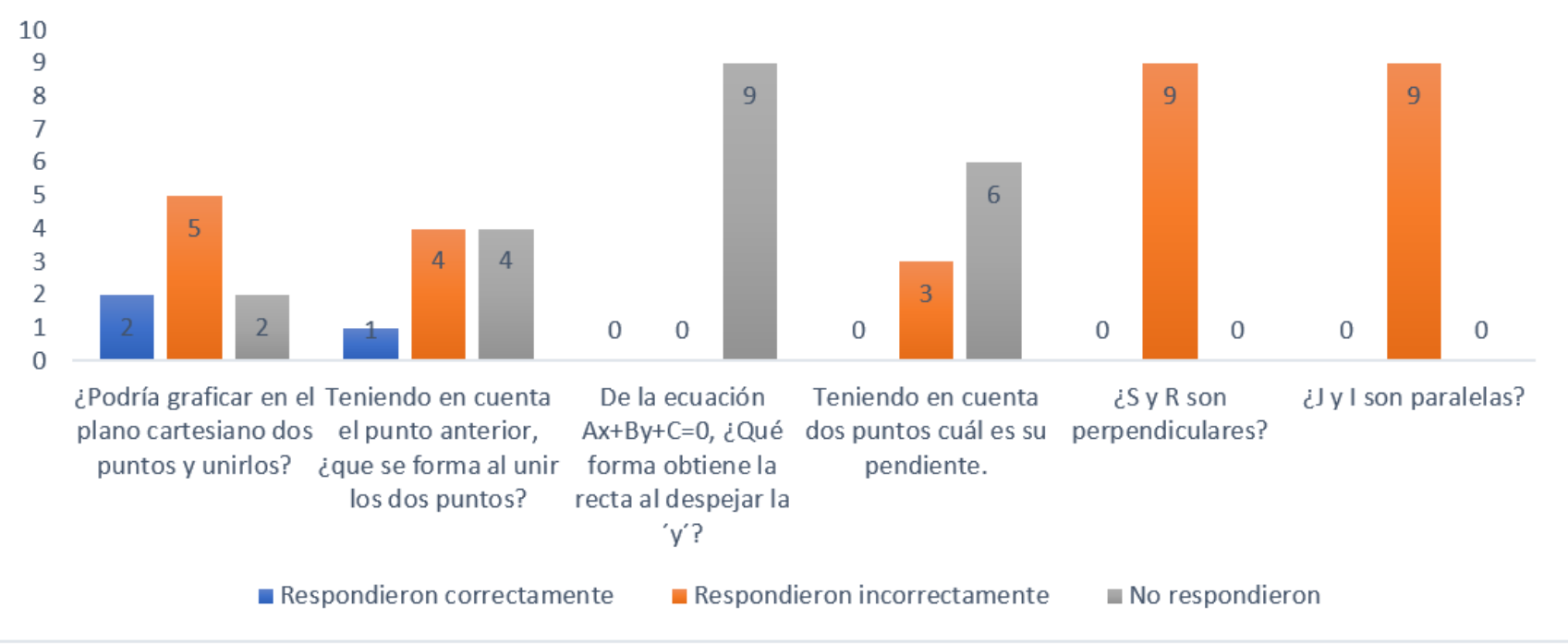

Figura 4. Respuesta de los estudiantes al test inicial.

Fuente: Los autores. 
Tabla 2: Análisis de la entrevista a los estudiantes y al docente de matemáticas.

\begin{tabular}{|c|c|c|}
\hline Entrevistado & Preguntas & Análisis \\
\hline Estudiantes & $\begin{array}{l}\text { - ¿Qué fue lo que más se les } \\
\text { dificultó al momento de } \\
\text { realizar el test? } \\
\text { - ¿Cuáles herramientas di- } \\
\text { gitales utilizó el docente } \\
\text { para enseñar la temática? }\end{array}$ & $\begin{array}{l}\text { Los estudiantes manifestaron que ya habían abordado la temática, pero no les } \\
\text { había quedado muy claro, porque el docente daba las clases, hacía un taller } \\
\text { y la evaluación, algunas veces no hacía retroalimentación de estas mismas. } \\
\text { Por otro lado, comentaron que el docente no utiliza herramientas educativas } \\
\text { digitales, pero agregaron que en la institución es complicado hacer uso de la } \\
\text { sala porque desde que inicia el año académico ya se encuentra apartado por } \\
\text { varios meses, no obstante, agregaron que les gustaría al menos dar una clase } \\
\text { donde se haga uso de las TICS. }\end{array}$ \\
\hline Docente & $\begin{array}{l}\text { ¿Cuáles herramientas } \\
\text { digitales utilizas o has } \\
\text { utilizado para enseñar } \\
\text { matemáticas? }\end{array}$ & $\begin{array}{l}\text { El docente de matemáticas manifestó que la institución cuenta con una } \\
\text { sala donde pueden ir e impartir las clases haciendo uso de las TICS, pero } \\
\text { la institución tiene más de seis cursos de décimo grado y para poder hacer } \\
\text { uso de esta tienen que informar con al menos una semana de anticipación, } \\
\text { además que es un trabajo arduo, porque a veces otros cursos hacen uso de } \\
\text { ella al mismo tiempo que él, entonces prefiere dar sus clases sin uso de estas } \\
\text { herramientas digitales. }\end{array}$ \\
\hline
\end{tabular}

Fuente: Elaboración propia con base a los resultados obtenidos en las entrevistas

Tabla 3: Análisis del test final (Análisis de la propuesta).

\section{Preguntas}

Sea $3 x+2 y-5=0$, escriba la línea recta en su forma canónica.

Halle la pendiente de los puntos $A(2,5) B(3,7)$

\section{Grafique la función}

$f(x)=3 x^{2}-2 x+7$ , grafíquelo en Geogebra y escriba una recta tangente a ella.

Realice el mismo proceso, pero con las siguientes cónicas:

$$
\text { - } x^{2}+y^{2}=9
$$

- $3 x^{2}-9 y^{2}=27$

Teniendo en cuenta el resultado del punto anterior, ¿qué puedes argumentar de la línea recta obtenida? ¿Podrías hallar más rectas tangente a $f(x)$.
Descripción

En esta punto los estudiantes debían aplica lo aprendido a través del programa Geogebra y escribir la línea recta en su forma canónica.

En este punto los estudiantes teniendo en cuenta el concepto de pendiente entre dos puntos, debía hallar el resultado.

En este punto, cada estudiante en su computador debía graficar la función y con base a esa función definida, hallarían una recta tangente.

En este punto y teniendo en cuenta el punto anterior, los estudiantes argumentarían el comportamiento de esta recta con base a la pendiente, además, a mover el punto a través de la recta observar que una función puede tener más de una recta tangente.
Análisis

Teniendo en cuenta la descripción de la pregunta, ocho estudiantes respondieron correctamente la pregunta, es decir el 89\% acertó, un estudiante cometió un error y fue de signo al dejar el punto de corte con el eje y negativo. Con base al test inicial, de dos estudiantes que respondieron correctamente este punto, hubo una mejora significativa.

Teniendo en cuenta la descripción de la pregunta, todos los estudiantes hallaron la pendiente de la línea recta, de igual manera que en punto anterior, y el test inicial, todos los estudiantes mostraron una mejora en su proceso de aprendizaje.

Teniendo en cuenta la descripción de la pregunta, el $78 \%$ de los estudiantes respondieron correctamente esta pregunta, al momento de revisar se pudo observar que el error de los otros estudiantes estuvo porque escribieron una función diferente a la planteada en el ejercicio, es decir, fue un error de observación, sin embargo, con respecto al test inicial hubo una mejora del $100 \%$.

Teniendo en cuenta la descripción de la pregunta, los estudiantes pudieron observar por medio del programa, al desplazar el punto a través de la función la recta tangente variaba e infirieron que en cualquier punto se podía hallar una recta siempre y cuando la pendiente existiera

Fuente: Elaboración propia con base a los resultados obtenidos en el test final 


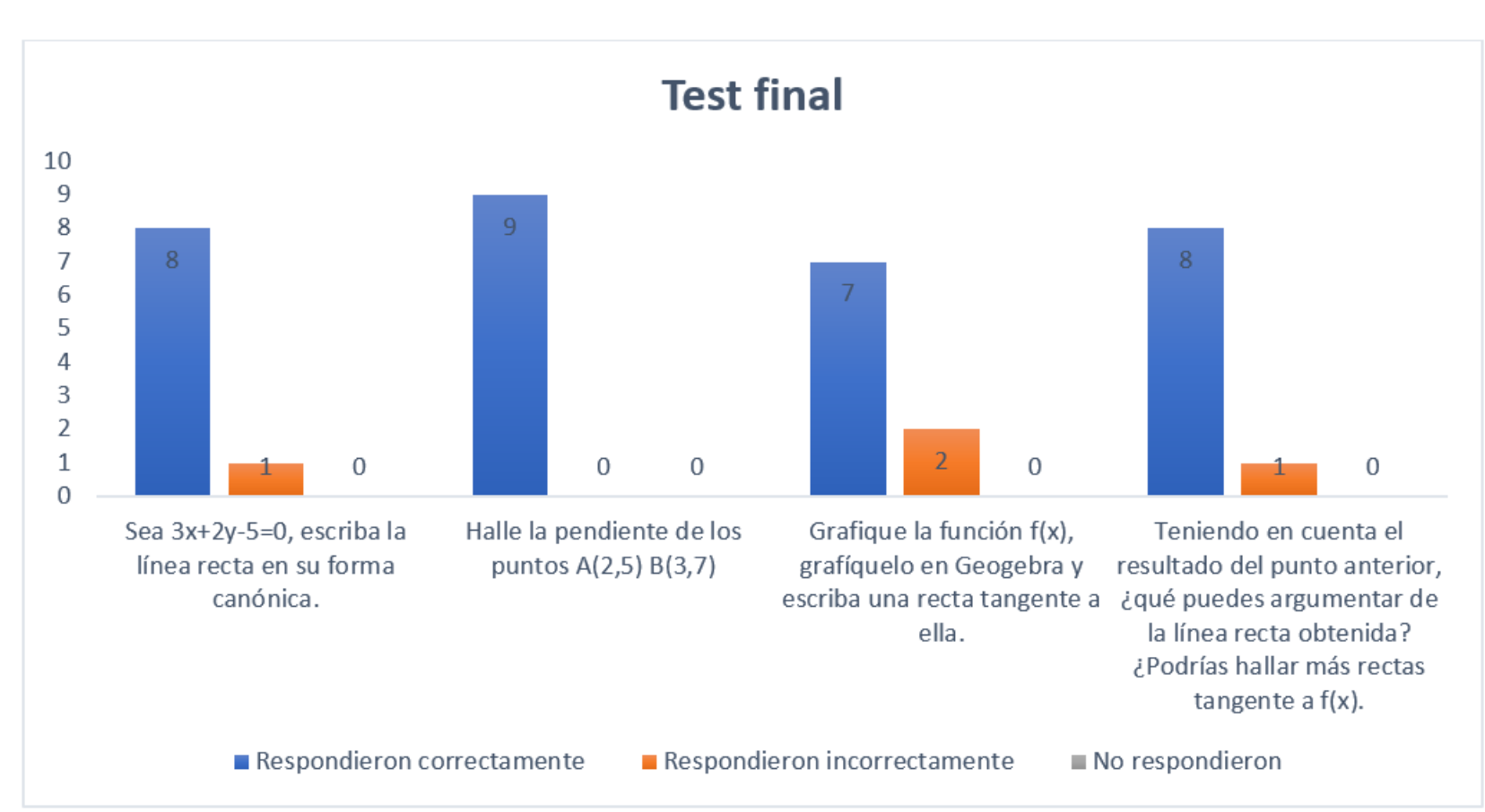

Figura 4. Análisis individual del Test Final.

Fuente: Los autores.

En la figura 3 , se puede observar el resultado del test final por pregunta. Con base a la gráfica, podemos observar que los estudiantes con respecto al test inicial mostrado en la figura 4, hubo una mejora significativa en el aprendizaje de los estudiantes cuando se implementó el uso del programa GeoGebra. De manera general el $89 \%$ de los estudiantes respondieron las preguntas correctamente, es decir que hubo una notable mejora con respecto al test inicial donde solo hubo un porcentaje de acierto del $5 \%$.

\section{Conclusiones}

El uso del software GeoGebra permitió de una u otra forma que los estudiantes modelaran situaciones geométricas de una manera más creativa y didáctica que como suele llevarse a cabo de manera tradicional, siendo relevante que este tipo de herramientas sea más utilizado en las aulas de clase, y más a sabiendas que los estudiantes con más necesidades requieren de estrategias por parte de los profesores que canalicen las mismas y fortalezcan los procesos.
Ante esto, es esencial que los profesores no solamente tengan conocimiento de los contenidos que se dispone a enseñar, sino también que sea capaz de relacionar lo que enseña con herramientas como GeoGebra, útiles en los procesos de enseñanza - aprendizaje de los estudiantes.

\section{Referencias}

Arias, R. (2013). Construcciones dinámicas con GeoGebra para el aprendizaje-enseñanza de la matemática. I Congreso de Educación Matemática de América Central y el Caribe: CEMACYC, Santo Domingo - Republica Dominicana.

Bejarano, M. (2017). Modelización matemática y GeoGebra en el estudio de funciones. una experiencia con estudiantes de ingeniería. Revista Ciencias de la Educación, 348.

Barahona, F., Barrera, O., Vaca, B., Hidalgo, B. (2015). GeoGebra para la enseñanza de la matemática y su incidencia en el rendimiento académico estudiantil. Revista Tecnológica ESPOL - RTE, 28(5), 121-132. 
Córdoba, Y., Ruiz, K.Y., Rendón, C.E. (2015). La comprensión del concepto de derivada mediante el uso de GeoGebra como propuesta didáctica. RECME, 1(1), 125-130.

Flórez, C., Yemail, C. (2017). Modelación y simulación con GeoGebra: una experiencia en el estudio de situaciones con medidas de área y volumen. Universidad Pontificia Bolivariana, Maestría en Ciencias Naturales y Matemáticas, Medellín - Colombia

Font, V. (1994). Motivación y dificultades de aprendizaje en matemáticas. Revista Suma, 92, 10-16.

Gruszycki, A., Oteiza, L., Maras, P., Gruszycki, L., Balles, H. (2012). Uso de GeoGebra para potenciar las diferentes representaciones en geometría analítica. Uso de Geogebra para potenciar las diferentes representaciones en geometría analítica. In Conferencia Latinoamericana de GeoGebra. (págs. 520-523). Uruguay.

Hernández- Sampieri, R. (2014). Metodolodía de la Investigación (6ta. ed.). McGraw-Hill / Interamericana Editores, S.A. de C.V, México.

Hernández-Sampieri, R; Fernández, C. Baptista, M. (2007). Metodología de la Investigación (5ta. ed.). McGraw-Hill / Interamericana Editores, S.A. de C.V, México.

Hitt, F. (2003). Dificultades en el aprendizaje del cálculo. Undécimo encuentro de profesores de matemáticas del nivel medio superior. Universidad Michoacana de San Nicolas de Hidalgo. Morelia.

Lehmann, C. (1989). Geometría analítica. Primera edición. México. Editorial Noriega Editores.

MaaB, K. (2007). Modeling in Class: What Do We Want the Students to Learn. Mathematical modelling: Education, engineering and economics, 63-78. https://doi. org/10.1533/9780857099419.2.63

MEN. (1998). Lineamientos Curriculares: Matemáticas. Bogotá: Magisterio.

MEN. (2013). Competencias TIC para el desarrollo profesional docente. Colombia aprende la red del conocimiento. Bogotá - Colombia
M.E.N. (2016). Derechos básicos de aprendizaje. Colombia

Pabón, J., Nieto, Z., Gómez, C. (2015). Modelación matemática y GeoGebra en el desarrollo de competencias en jóvenes investigadores. Revista Logos Ciencia y Tecnología, 7(1), 6570. https://doi.org/10.22335/rlct.v7i1.257

Padilla, I., Valbuena, S., Rodríguez, E. (2018). El juego y la inteligencia lógico-matemática de estudiantes con capacidades excepcionales. Educación y Humanismo, 20(35), 166-183. https://doi.org/10.17081/eduhum.20.35.2964

Padilla, I., Mayoral, V. (2020). Las tutorías académicas en el fortalecimiento del álgebra en estudiantes de octavo grado en una escuela distrital de Barranquilla. Zona Próxima, 32, 33-54.

Ramírez, M., Oktac, A. García, C. (2006). Dificultades que presentan los estudiantes en los modos geométrico y analítico de sistemas de ecuaciones lineales. Campo de investigación: Pensamiento algebraico; Nivel educativo: Superior. 6, p.p. 413-418.

Sáenz, E; Patiño, M., Robles, J. (2017). Desarrollo de las competencias matemáticas en el pensamiento geométrico, a través del método heurístico de Pólya. Panorama, 11(21), 53-67.

Villa- Ochoa, J., Ruíz, H. (2009). Modelación en educación matemática: una mirada desde los lineamientos y estándares curriculares colombianos. Revista Virtual Universidad Católica del Norte, 27, 1-21.

Villa-Ochoa, J., Rojas, C., Cuartas, C. (2010). ¿Realidad en las matemáticas escolares?: reflexiones acerca de la "realidad" en modelación en educación matemática. Revista Virtual Universidad Católica del Norte, 29, 1-17. https://doi.org/10.4067/ $\underline{\text { S0718-50062018000200025 }}$

Villa-Ochoa, J.A., González-Gómez, D., Carmona-Mesa, J.A. (2018). Modelación y Tecnología en el Estudio de la Tasa de Variación Instantánea en Matemáticas. Formación universitaria, 11(2), 25-34. 
Villa-Ochoa, J.A. (2015). Modelación matemática a partir de problemas de enunciados verbales: un estudio de caso con profesores de matemáticas. Magis Revista Internacional De Investigación En Educación, 8(16), 133-148. https:// doi.org/10.11144/Javeriana.m8-16.mmpe

Verschaffel, L., De Corte, E. (1997). Teaching Realistic Mathematical Modeling in The Elementary School: A Teaching Experiment with
Fifth Graders. Journal for Research in Mathematics Education, 28(5), 577-601. https://doi. org/10.5951/jresematheduc.28.5.0577

Zaldívar, J., Medina, G., Kakes, A. (2018). Modelación y tecnología en la enseñanza de las matemáticas. En Arturo, Luis; Páges, Daniela (Eds.), Acta Latinoamericana de Matemática Educativa (pp. 954-961). México, DF: Comité Latinoamericano de Matemática Educativa. pp (954-961). 